\title{
Uma proposta da eficiência do uso da Metodologia Ativa Baseada em Problemas, utilizando Dojo de Programação, aplicada na disciplina de Lógica de Programação
}

\author{
Andreza Bastos Mourão ${ }^{1,2}$ \\ ${ }^{1}$ Universidade do Estado do Amazonas (UEA) \\ Av. Darcy Vargas, 1200. Manaus - AM - Brasil. \\ ${ }^{2}$ Centro Universitário do Norte (UNINORTE) \\ Rua Huascar de Figueiredo, n ${ }^{\circ}$ 290, Centro. Manaus - AM - Brasil. \\ amourao@uea.edu.br, andreza.mourao@uninorte.com.br
}

\begin{abstract}
This paper describes the experience of an educational project teaching at the Programming Logic course using active learning methodology based on problems and Dojo programming method. The practical experiences of this project are motivating and have contributed positively to the better performance of the students in the discipline, reducing evasion rates, improving communication in pairs and in groups, developing the ability to divide tasks and solve problems, among others. The article aims to share the adopted methodology, in order to stimulate teachers and students in the use and practice of this method and innovative teaching environment.
\end{abstract}

Resumo. Este artigo descreve a experiência de um projeto de ensino voltado para a disciplina de Lógica de Programação, utilizando a Metodologia Ativa de Aprendizagem Baseada em Problemas e do Método de Programação Dojo. As experiências práticas deste projeto são motivadoras e contribuíram positivamente para o melhor desempenho dos alunos na disciplina, a diminuição dos índices de evasão, melhorar a comunicação em pares e em grupo, desenvolver a habilidade em dividir tarefas e resolver problemas, entre outros. $O$ artigo visa compartilhar a metodologia adotada, a fim de estimular docentes e discentes no uso e prática deste método e ambiente de ensino inovador.

\section{Introdução}

Segundo Piekarski et al (2015), Programação de Computadores é um dos conteúdos mais complexos da área de Computação, constituindo uma das bases mais importantes para a futura atuação profissional dos estudantes. O grau de dificuldade desses conteúdos se reflete na quantidade de desistências ao longo da disciplina e no alto índice de reprovação.

Ministrar aulas de Lógica de Programação em Instituições de Ensino (IES), requer do professor o uso de métodos, técnicas, ferramentas, e habilidades dinâmicas e inovadoras que venham a somar no processo de ensino e aprendizagem. A aprendizagem que envolve a motivação e novas formas de ensino, alcançando as dimensões afetivas e intelectuais, tendem a ser mais duradoura e sólida, aproximando professor e aluno em uma aprendizagem colaborativa. Os cursos de Computação do Ensino Superior incluem 
VI Congresso Brasileiro de Informática na Educação (CBIE 2017)

Anais do XXIII Workshop de Informática na Escola (WIE 2017)

disciplinas relacionadas a Algoritmos e Programação de Computadores, as quais promovem o desenvolvimento de habilidades essenciais para o bom rendimento do estudante e do regresso desses cursos [Souza, 2016].

A Metodologia Ativa possibilita uma leitura e intervenção consistente sobre a realidade, e valoriza todos os atores no processo de construção coletiva e seus diferentes conhecimentos, além de promover a liberdade no processo de pensar e no trabalho em equipe.

O aprendizado baseado em problemas resulta do processo de trabalhar através da compreensão ou resolução de um problema. O ponto de partida para o aprendizado é o problema [Attle e Baker, 2007]. Esta metodologia é bastante utilizada no ensino da Lógica de Programação, por perceber que quanto mais prática o aluno tiver, em analisar, compreender e encontrar a resolução adequada para o problema, mais motivado e melhores resultados este irá obter.

Integrar uma metodologia ativa e uma técnica de programação, é uma proposta que vem trazendo resultados neste campo, cuja dificuldade apresentada pelos alunos é imensa, em consequência do ato de pensar, raciocinar, refletir, e tomar a decisão mais adequada para resolver o problema. Assim como, adquirir e gerenciar conhecimentos específicos de algoritmos que envolvem: sintaxe, construção de blocos, estruturas de controle, variáveis, tipos de dados e outros conceitos que são necessários para que a máquina possa interpretar e executar o que foi programado.

Este artigo apresenta uma experiência prática no processo de ensino e aprendizagem da disciplina de Lógica de Programação (geralmente oferecida nos $1^{\circ}$ e $2^{\circ}$ período das IES nos Cursos de Computação), utilizando como ferramenta metodológica a combinação da Metodologia Ativa: Aprendizagem Baseada em Problemas (ABP) e a técnica de programação Coding Dojo, no processo de construção de algoritmos. O objetivo desse trabalho é relatar a experiência no aprendizado e o desempenho obtido pelos alunos de graduação em Computação, no contexto da disciplina de Lógica de Programação, ministradas em IES pública e particular. Ao apresentar a proposta e os resultados desse projeto, espera-se contribuir para a criação de um ambiente de ensino motivador, tornando o processo de aprendizagem mais dinâmico, competitivo, colaborativo e prazeroso. E com isso, incentivar as IES e docentes da área a adotar combinações de metodologias de ensino ativas, dentre elas a Aprendizagem Baseada em Problemas, unificando métodos e técnicas aplicadas no mercado de trabalho, em particular nas empresas de desenvolvimento de software.

Este trabalho apresenta-se dividido em seções: após a atual Seção de Introdução, a Seção 2 descreve a Metodologia científica adotada, a Seção 3 descreve a Metodologia Ativa Baseada em Problemas com o método Dojo de Programação. Na Seção 4 são descritos e discutidos os Resultados apresentados e por fim na Seção 5 são relatadas as Considerações finais.

\section{Metodologia Científica}

A metodologia definida para a utilização da Metodologia Ativa ABP com a técnica Dojo de Programação foi especificada em três etapas, conforme descrito a seguir:

1 - Planejamento: etapa responsável pela definição das tarefas, cronograma, equipes, da Metodologia Ativa ABP e do uso do Método Conding Dojo. 
VI Congresso Brasileiro de Informática na Educação (CBIE 2017)

Anais do XXIII Workshop de Informática na Escola (WIE 2017)

2 - Desenvolvimento: etapa responsável pela condução dos conteúdos, aplicação de problemas e uso do método em duas etapas específicas, consistindo num experimento prático.

Nesta fase a turma é orientada sobre a dinâmica, tempo e atuação dos alunos durante o processo. Neste momento, os alunos já escrevem algoritmos, já realizam testes de mesa e já atuam em grupo na resolução dos problemas propostos. O uso de uma ferramenta de apoio é essencial para o sucesso do processo.

3 - Avaliação do Desempenho dos Alunos: nesta etapa é apresentado os resultados do projeto. Os alunos foram avaliados por meio de nota, visando assim obter uma média final constituída da parte teórica e prática. As planilhas com as respectivas notas dos alunos foram consideradas a fim de se obter os dados quantitativos a serem expressados por meio de gráficos e assim comparados entre as IES. Houve a aplicação de um questionário (avaliação qualitativa) para a obtenção da opinião dos estudantes sobre a inserção e uso do método durante o processo de ensino de Lógica de Programação.

\section{Metodologias Ativas}

De acordo com Christensen et al (1991), quando o objetivo é o pensamento crítico ou a resolução de problemas, tanto professores quanto alunos devem modificar seus papeis e responsabilidades tradicionais. A aprendizagem ativa é o processo em que os estudantes se tornam ativamente envolvidos em seu próprio aprendizado, realizando suas próprias descobertas. Neste sentido, de acordo com Akili et al (2011), a aprendizagem ativa requer atividades de aprendizagem significativa em grupos supervisionados por um professor experiente.

As Metodologias Ativas se constituem de processos interativos e que estimulam a autoaprendizagem, onde o professor atua como orientador e o aluno como sendo o agente central do processo. Os princípios que norteiam esta metodologia, estão relacionados com a aprendizagem construtiva, colaborativa, interdisciplinar, contextualizada, reflexiva, crítica, investigativa, humanista, motivadora e desafiadora.

As Metodologias Ativas envolvem alguns métodos, tais como: Aprendizagem Baseada em Problemas, Problematização, Estudo de Caso, Aprendizagem Baseada em Projetos, Discussão em Classe, Seminários, Mapas Mentais e Conceituais, Aprendizagem Baseada em Grupos e Simulação.

\subsection{Aprendizagem Baseada em Problemas (ABP)}

A Aprendizagem Baseada em Problemas (ABP) ou de origem inglês Problem Based Learning $(P B L)$ é uma Metodologia Ativa de ensino-aprendizagem que surgiu na escola de Medicina da Universidade McMaster, no Canadá, em meados dos anos 1960. A ABP é um processo de aprendizagem em que sua fundamentação pressupõe uma construção de significados, e não um simples processo de recepção de informações, estando presente em diversas pesquisas da psicologia cognitiva.

Conforme relata Prince (2004) Problem Based Learning é um método instrutivo onde um problema relevante é introduzido no início do ciclo instrucional e usado para fornecer contexto e motivação para o aprendizado que segue. $O$ processo de aprendizagem do ABP faz com que o aluno modifique sua relação com o mundo, fazendo com que este ultrapasse a vontade de aprender. Quando se fala de aprendizagem, o sujeito 
VI Congresso Brasileiro de Informática na Educação (CBIE 2017)

Anais do XXIII Workshop de Informática na Escola (WIE 2017)

que aprende é o centro do processo, e isso implica em mudanças na forma de pensar e de se relacionar com o mundo.

A ABP é uma metodologia que difere do modelo tradicional de ensino, pelo fato de utilizar problemas da vida real para iniciar o processo de aprendizagem, visando estimular o desenvolvimento de habilidades para solucionar problemas [Oliveira 2012]. A abordagem consiste em apresentar um ou vários problemas e solicitar uma solução. Esta solução terá que envolver os conteúdos já trabalhados até o momento, começando do mais simples e podendo gradativamente aumentar o grau de complexidade. Esta aprendizagem consiste em esclarecer termos e expressões do texto, definir o problema por meio de questões, analisar o problema, levantar e formular hipóteses, se necessário procurar informações, responder o problema e discutir conhecimentos.

\subsection{Método de ensino Coding Dojo - Dojo de Programação}

A palavra "Dojo" (lê-se dojô) é uma palavra de origem japonesa oriunda das artes marciais. Os Dojos são locais onde estudantes de luta se reúnem para realizar competição, treinamentos, desafios ou testes que possibilitam a mudança de faixa. E neste momento, são realizados vários: "Kata" (nome dado as sequências precisas de movimentos de luta), utilizadas para exercitar técnicas de defesa. Os Dojos são locais de treinos de artes marciais, onde dois lutadores praticam e os demais aprendem observando e revezando-se no tatame.

Dojo de Programação, em inglês Coding Dojo, é uma atividade que vem sendo usada por programadores para praticar métodos ágeis, especialmente em desenvolvimento dirigido por testes [Beck e Andres 2004], programação pareada [Williams and Kessler 2002] e passos de bebê [Beck e Andres 2004]. Os Dojos de Programação são oriundos da técnica japonesa, porém com enfoque na programação. $\mathrm{Na}$ maioria dos casos são promovidos em empresas de desenvolvimento, onde grupos de desenvolvedores se reúnem para fazer networking e compartilhar conhecimentos, favorecendo assim o aprendizado voluntário e colaborativo.

Um Dojo deve promover um ambiente divertido e cooperativo, sem enfoque na competição, e sim na colaboração, no compartilhamento, aprimoramento, no aprendizado, na troca de informações, melhorias e qualidade de código, promovendo assim um networking na programação. É um ambiente inclusivo, colaborativo e seguro para testar novas ideias, criar estratégias, identificar problemas no código, sugerir, contribuir e melhorar a comunicação entre a equipe de desenvolvedores. Apresentandose como um método excelente para ensinar Lógica de Programação, Linguagem de Programação, Estrutura de Dados, Programação Orientada a Objetos, Análise de Algoritmos, dentre outras disciplinas que envolvam a Programação de Computadores.

Um Dojo de Programação na prática é um encontro de profissionais da área da computação, que se reúnem para treinar e aprender, resolvendo problemas (desafios) computacionais. É um networking, onde os participantes estão buscando melhorar suas habilidades técnicas, colaborar em equipe e programar em pares. A sessão é orientada por um dos profissionais, e os demais viram temporariamente plateia, que observam o desenvolvimento e colaboram no momento oportuno. No final, são discutidas as lições aprendidas, as estratégias, os pontos positivos e negativos do evento e os pontos de melhorias. Um dos diferenciais desta prática são os passos de bebê, "Baby Steps" em 
VI Congresso Brasileiro de Informática na Educação (CBIE 2017)

Anais do XXIII Workshop de Informática na Escola (WIE 2017)

inglês, é o nome dado a técnica de fazer pequenas mudanças na direção da solução do problema [Beck and Andres 2004].

Para montar uma sessão de Dojo de Programação, torna-se necessário uma sala ampla e confortável para os participantes, um telão, um projetor e um computador. Todos os aparatos tecnológicos testados e funcionando, para que os participantes possam desempenhar suas funções e visualizar tudo o que for realizado por meio do projetor.

Existem várias dinâmicas, ou formatos relacionados aos Coding Dojos, alguns dos mais utilizados, pode ser observado na tabela 1 a seguir.

Tabela 1. Métodos Coding Dojo.

\begin{tabular}{|c|c|}
\hline \multicolumn{2}{|r|}{ CODING DOJO } \\
\hline MÉTODOS & DESCRIÇÃO \\
\hline RANDORI & 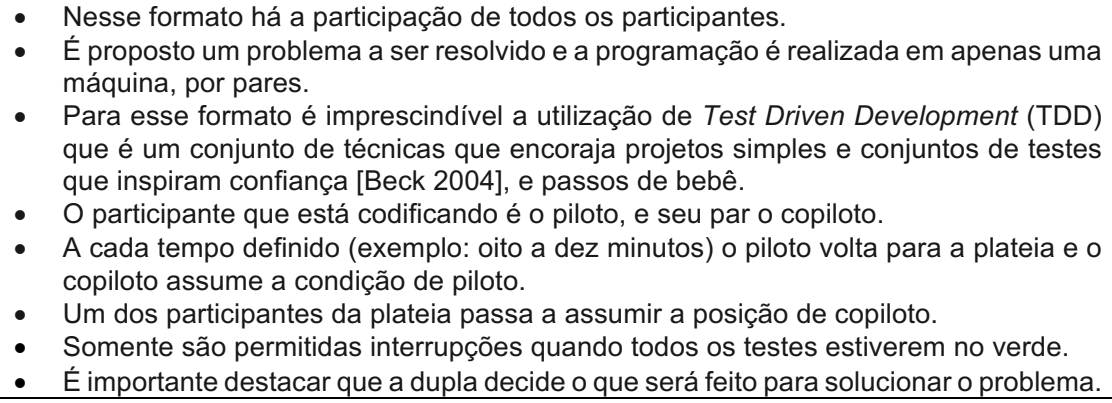 \\
\hline KATA & $\begin{array}{l}\text { - Nesse formato existe a figura do apresentador. } \\
\text { - O apresentador demonstra uma solução pronta, previamente desenvolvida. } \\
\text { - O objetivo é que todos os participantes sejam capazes de reproduzir a solução } \\
\text { alcançando o mesmo resultado, sendo permitido realizar interrupções para sanar } \\
\text { dúvidas a qualquer momento; }\end{array}$ \\
\hline KAKE & $\begin{array}{l}\text { - É um formato semelhante ao Randori, porém há várias duplas trabalhando } \\
\text { simultaneamente. } \\
\text { - A cada turno as duplas são trocadas, promovendo a integração entre todos os } \\
\text { participantes do evento. } \\
\text { - Nesse formato é necessário um conhecimento mais avançado dos participantes. }\end{array}$ \\
\hline
\end{tabular}

Os formatos permitem aos programadores serem desafiados, com novos problemas e linguagens, buscando sempre novas e melhores soluções. Melhorando assim, o ambiente da empresa ou da organização que a utiliza.

\section{Metodologia Ativa de Aprendizagem Baseada em Problemas utilizando o método Coding Dojo}

Barbosa et al (2011), descrevem que a aprendizagem inicial de programação é considerada um processo em que os alunos enxergam como um verdadeiro teste de vocação. Os alunos que têm pouco ou nenhum sucesso nesse processo, geralmente o abandonam e começam a olhar para outras possibilidades, às vezes, até para a mudança de carreira. Por este motivo, o índice de reprovação e evasão nesta disciplina ainda se apresenta bastante elevado.

Ser professor de disciplinas de programação sejam nas turmas iniciais, intermediárias ou finais, é um papel que exige o domínio, técnica e conhecimento de métodos de ensino, que somados aos conteúdos ministrados, possam elevar a atenção, colaboração e participação dos alunos. Portanto, utilizar tais requisitos, contribui para minimizar o baixo rendimento do desempenho do aluno e a evasão destes nas disciplinas, ocasionadas em grande parte pela complexidade de conceitos e estruturas. 
O uso de Metodologias Ativas aplicadas no processo de ensino e aprendizagem, contribuem para a formação do estudante, e aliadas a métodos que trazem resultados evidenciados, podem resultar no elevado índice de satisfação de professores e desempenho de estudantes. O Dojo de Programação é um método que somado a ABP traz resultados expressivos ao processo de ensino e aprendizagem. Os requisitos necessários para a aplicação do Dojo dependem:

1. Do conhecimento prévio dos alunos em relação ao conteúdo abordado;

2. Da resolução prévia de problemas a serem explorados no evento;

3. Da definição da quantidade e dos participantes de cada equipe, assim como sua interação e colaboração, no que diz respeito a resolução de exercícios;

4. De um ambiente favorável, para que a realização seja bem-sucedida;

5. Da clara explicação do objetivo, funcionamento e das regras antes do evento;

6. Da seleção e organização dos recursos tecnológicos a serem utilizados;

7. Do professor gerenciando e conduzindo a dinâmica, visando transmitir tranquilidade, responsabilidade, organização, intermediação e participação dos estudantes.

Os passos acima são fundamentais para se atingir o objetivo desejado que é elevar o índice de aproveitamento e aprendizagem de programação na prática, efetivando o que foi visto de forma teórica e por meio de conceitos e da resolução de problemas em sala de aula.

\subsection{Aplicação prática}

A Metodologia foi aplicada nas IES Centro Universitário do Norte (UNINORTE) e Universidade do Estado do Amazonas (UEA), a primeira de categoria particular e a segunda pública. A metodologia foi aplicada com as turmas da Computação: Licenciatura em Informática, Engenharia da Computação, Ciência da Computação e Tecnologia em Análise e Desenvolvimento de Sistemas.

No primeiro dia de aula o professor apresenta para os alunos a ementa da disciplina, e comenta que utilizará a Metodologia Ativa ABP em paralelo com a técnica Dojo de Programação. A disciplina de Lógica de Programação nas IES as quais foram aplicadas o experimento possuem os seguintes conteúdos em sua ementa: conceitos básicos (lógica, variáveis, constante, operadores aritméticos, relacionais e lógico) e estruturas de controle (sequencial, condicional e de repetição).

O formato do Dojo utilizado foi o método Randori, por ser o mais adequado a proposta de ensino das IES, em paralelo com a Metodologia Ativa ABP. Neste formato foi possível contar com a participação de toda a turma, que foi dividida em grupos de 5 a 6 alunos em função da grande quantidade de alunos matriculados. As equipes foram submetidas a resolução de $1(\mathrm{um})$ problema sorteado de uma lista de exercícios que vinham sendo trabalhadas em sala de aula. Cada sessão tem duração de 8 a 10 minutos, dependendo da complexidade dos exercícios. O conteúdo trabalhado refere-se a estrutura de controle condicional e de repetição. A resolução é observada por toda a classe.

Para a realização da atividade foi escolhida a sala de aula como o ambiente ideal, composto de um computador (terminal), um projetor, um quadro com anotações referentes ao evento e informativos. Podendo ser utilizado a ferramenta de apoio, em conformidade com a ementa da disciplina. Neste sentido, por se tratar da disciplina de 
VI Congresso Brasileiro de Informática na Educação (CBIE 2017)

Anais do XXIII Workshop de Informática na Escola (WIE 2017)

Lógica de Programação, foi escolhida o uso da ferramenta VisuAlg 2.5, para apoiar a codificação dos algoritmos (portugol), validar o código e realizar o teste de mesa. A Figura 1, apresenta o ambiente de sala de aula e alunos participantes.

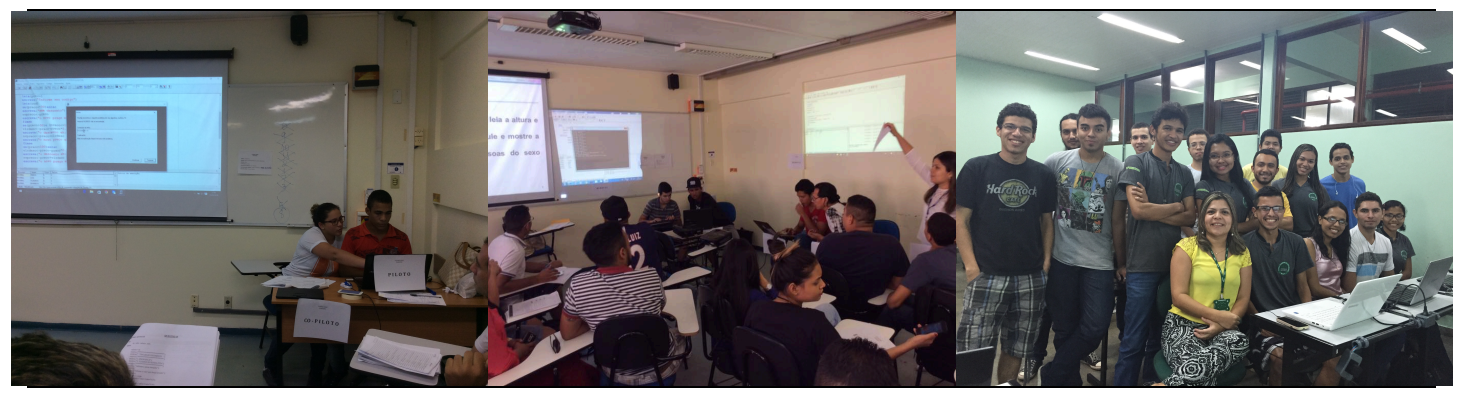

Figura 1. Ambiente adequado para a aplicação do Coding Dojo.

Na Figura 1, é possível observar o par de alunos que iniciam a atividade de programação, onde um assume o papel de piloto e o outro de copiloto. A programação em par é uma técnica caracterizada por uma dupla sentada lado a lado compartilhando o mesmo computador e desenvolvendo a solução colaborativamente. É um diálogo e programação simultânea entre duas pessoas [Beck and Andres 2004]. Essa técnica permite que os programadores mantenham a atenção na tarefa, alinhem as mensagens do sistema, esclareçam ideias, e quando alguém não consegue seguir em frente o outro toma iniciativa e ambos se mantém alinhados com as práticas do time [Beck and Andres 2004].

Todos os alunos participam da sessão, o tempo de 2 (dois) minutos é alternado, cada aluno participante assume o papel de piloto e copiloto. A equipe é responsável pela definição das estratégias mais adequadas, de troca de alunos, de código (estrutura e sintaxe) entre outras. No final, a equipe explica para turma o que foi realizado, suas estratégias, as dificuldades encontradas e a experiência vivida.

O aprendizado cooperativo é constituído por técnicas de ensino em que os estudantes trabalham em atividades de aprendizado em pequenos grupos e recebem gratificações e reconhecimento baseado no desempenho do grupo [Slavin 1980]. A ideia é ampliar a troca de informações e conhecimentos entre os estudantes para motivar seu próprio aprendizado e estimular uns aos outros [Slavin 1980], [Attle e Baker 2007], [Burguillo 2010].

\section{Resultados e Discussões}

Esta proposta tem como objetivo da Aprendizagem, propiciar ao aluno a assimilação de um grande volume de conteúdo, por meio de uma aprendizagem significativa e em grupo, permitindo que os mesmos passem a tomar decisões em conjunto, melhorando o relacionamento interpessoal, e se sentindo mais seguros e confiantes, além de melhorar suas expressões oral e escrita, e estimulando suas habilidades em resolver problemas e conduzir projetos.

Em uma das turmas onde o Dojo foi aplicado havia um aluno Portador de Necessidades Educacionais Especiais (PNEEs), identificada como Deficiência Auditiva, este assistia as aulas acompanhado de intérprete de LIBRAS. Desta forma, durante as aulas o que era ensinado pelo professor, era transmitido para o aluno. $\mathrm{O}$ aluno teve um destaque e um papel significativo, conseguindo identificar e apontar o erro no código, por meio da observação na sintaxe, contribuindo para o sucesso da equipe. 
Os alunos antes do evento, já haviam realizado atividades em sala de aula (em equipe e mediada pelo professor) e extraclasse, por meio de lista de exercícios. Após o evento do Dojo de Programação, foi realizada uma reunião onde foram destacados os pontos positivos e negativos do uso do método, e foram constatados os benefícios, as deficiências, as dificuldades encontradas, as estratégias utilizadas pelas equipes, a liderança, dentre outros itens significativos, deixando os alunos motivados para posteriores competições de Dojo oferecidas nas IES.

Os benefícios identificados, durante e após a aplicação deste método foram:

- A estimulação da Programação em Par: piloto e copiloto, colocam a mão na massa, digitando, identando código e realizando testes. A função do copiloto é palpitar e ajudar olhando e apontando onde pode melhorar, e isso se repete a cada novo tempo, quando ocorre a troca do copiloto.

- O desenvolvimento de tarefas de baixo, médio e alto nível de complexidade. Assim como a prática de análise, síntese e auto avaliação.

- A exercitação da inteligência (funções de pensar, observar, raciocinar, refletir, entender, se questionar e outros).

- A resolver problemas, estimulando o aprendizado, visando atingir metas e resultados. Pensando em estratégias e tomando as decisões mais adequadas e em grupo.

- Alunos confiantes, desenvolvendo suas competências e habilidades com satisfação e orgulhosos.

As dificuldades encontradas foram o melhor domínio da sintaxe, conflitos de ideias (uso da lógica) e a tomada de decisões sobre pressão. As atividades também foram realizadas entre turmas, em uma aula agendada no sábado (Figura 1).

Foram promovidas competições de Dojo de Programação na IES, onde os alunos formaram equipes e competiram entre si. $\mathrm{O}$ evento resultou em um networking, interação e colaboração entre cursos e turmas da computação. A Figura 2 mostra as equipes vencedoras dos eventos, no decorrer dos dois últimos anos na IES particular UNINORTE.

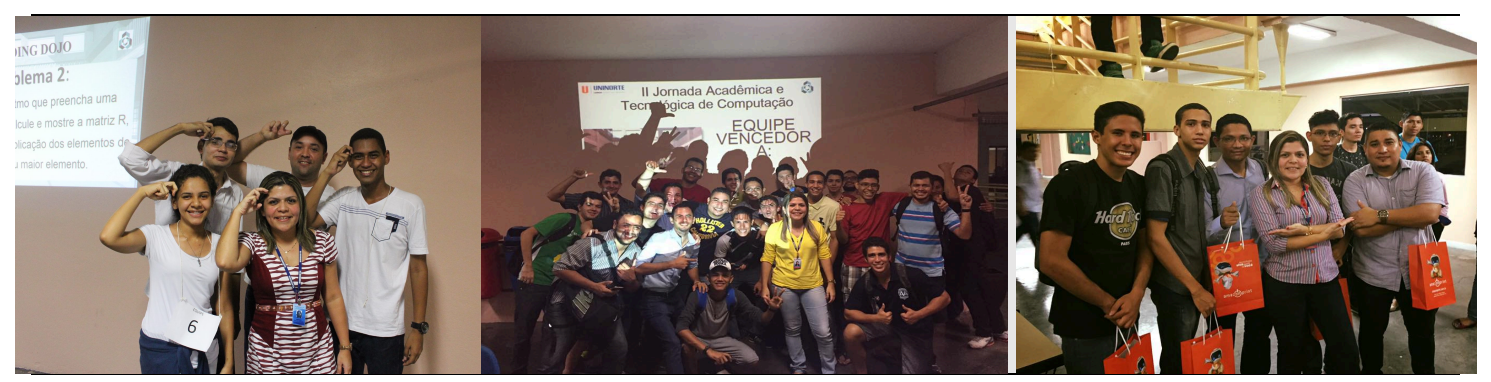

Figura 2. Evento Dojo de Programação (UNINORTE)

Neste evento percebeu-se que as turmas vencedoras da competição, se destacaram pelo tempo, código (sintaxe), cooperação, colaboração, uso de estratégias e tomadas de decisões adequadas e estavam preparadas para trabalhar sob pressão. A primeira equipe vencedora dedicou a sua vitória ao colega deficiente auditivo, conforme pode ser observado na Figura 2. 
VI Congresso Brasileiro de Informática na Educação (CBIE 2017)

Anais do XXIII Workshop de Informática na Escola (WIE 2017)

\subsection{Analise do índice de Aprovação das Turmas utilizando o Dojo de Programação}

Realizada a Avaliação Diagnóstica do Desempenho dos Alunos, os resultados foram mensurados por meio das planilhas de notas e frequência para a obtenção dos dados quantitativos significativos relacionados com a inserção e uso do método durante o processo de ensino e aprendizagem. A Figura 4, apresenta o resultado expresso em percentual relativo ao desempenho das turmas de ambas as IES.
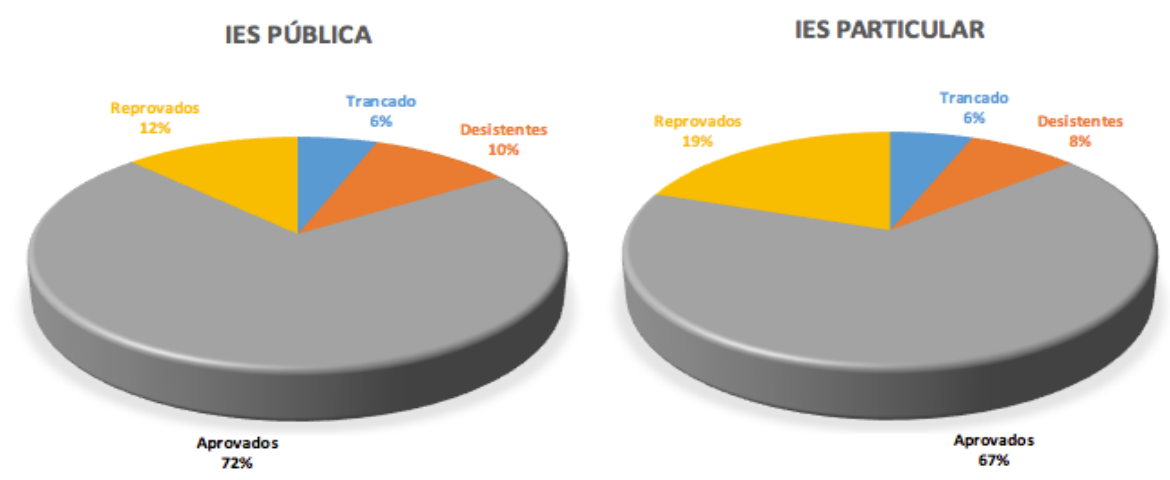

Figura 4. Percentual de Aprovação com o uso da Metodologia.

A avaliação do desempenho dos alunos com o uso do método, mostra que vem ocorrendo um aumento gradativo e significativo no índice de alunos aprovados na disciplina de Lógica de Programação, principalmente quando comparadas com planilhas anteriores ao uso da metodologia. Os dados gerados são realizados a cada final de semestre, visando obter um feedback das turmas. Percebeu-se que antes do uso do método apenas 40 a $45 \%$ dos alunos eram aprovados na disciplina e com o uso do método temos 67 a $72 \%$ de aprovação, considerando os casos de trancamentos, desistência e reprovação. A Avaliação qualitativa realizada refere-se a opinião dos estudantes realizadas por meio da aplicação de questionários, antes e depois do uso do método. A Figura 5 apresenta os resultados.

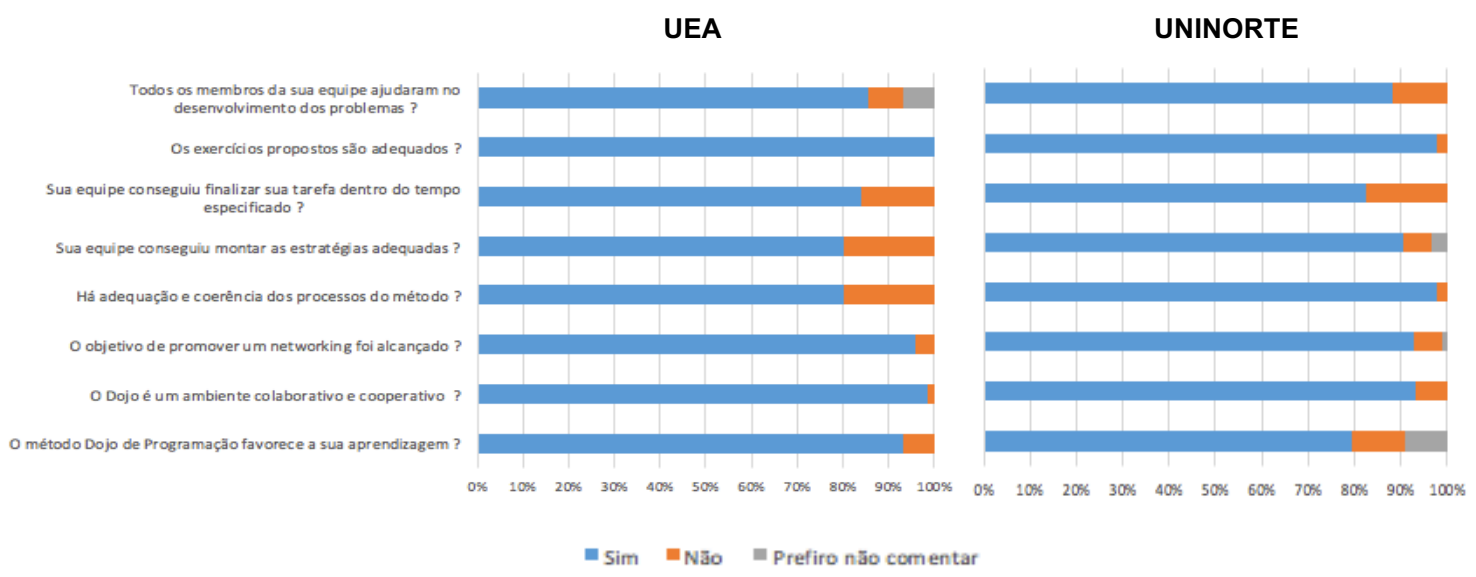

Figura 5. Avaliação dos Estudantes - Metodologia (IES pública/particular).

Nesta avaliação de desempenho, 75 respostas de alunos da IES pública foram computadas, dentre um universo de 89 alunos, nos quais 14 são desistentes ou tiveram suas matrículas trancadas. Das IES particulares, 88 respostas foram computadas, dentre um universo de 102 alunos, nos quais 14 são desistentes ou tiveram suas matrículas trancadas. Os resultados foram satisfatórios, revelam que os alunos apontam que o 
VI Congresso Brasileiro de Informática na Educação (CBIE 2017)

Anais do XXIII Workshop de Informática na Escola (WIE 2017)

método contribui para a aprendizagem em 80 a 90\%. Mostra que o ambiente é colaborativo e cooperativo 90 a $98 \%$, bem como promove o networking. Relata que ainda é necessário aprimorar as estratégias e a atenção ao tempo disponível para a atividade, oriundos da prática. Houve cooperação e participação da equipe em $80 \%$ aproximadamente e 98 a $100 \%$ apontam os exercícios propostos adequados.

\section{Conclusão}

O Dojo de Programação é um ambiente de desenvolvimento inovador para testar novas ideias, promover o networking e compartilhamento entre os membros da equipe, tornar a aprendizagem colaborativa, estimular a cooperação, a programação em pares e desafiar programadores com novos problemas e novas linguagens. A avaliação da prática realizada, nos faz refletir professor e alunos a importância da aprendizagem ativa, que se caracteriza quando ocorre a interação do aluno com o assunto em estudo, onde o mesmo é estimulado a construir o conhecimento por meio de suas práticas fundamentadas pela teoria abstraída. Nesse momento, ocorre a valorização, comunicação e colaboração entre os colegas, o estímulo da memória, a aprendizagem competitiva, a busca pela resposta correta, a análise, o questionamento e a solução de problemas. Os alunos quando motivados buscam atingir seu objetivo vencendo as adversidades encontradas por meio das soluções mais adequadas.

\section{Referência}

Attle, S. and Baker, B. (2007). Cooperative learning in a competitive environment: classroom applications. International Journal of Teaching and Learning in Higher Education, 19(1):77-83.

Akili,W. (2011). On implementation of problem-based learning in engineering education: Thoughts, strategies and working models. In Proceedings of the 2011 Frontiers in Education Conference, FIE '11, Washington, DC, USA. IEEE Computer Society.

Barbosa, Lucinéia, and Marcia Gonçalves De Oliveira (2011). Metodologia ANEA Para Avaliação Online de Lógica de Programação. SBIE (2011): 1394-1397.

Beck, K. and Andres, C. (2004). Extreme programming explained: embrace change (2nd edition). Addison-Wesley Professional.

Burguillo, J. C. (2010). Using game theory and Competition based Learning to stimulate student motivation and performance. Computers \& Education, 55(2):566-575.

Christensen, C. R., Garvin, D. A., and Sweet, A, (1991). Education for Judgment: The Artist of Discussion Leadership. Harvard Business School Press.

Piekarski, Ana Elisa Tozetto, Miazaki, Mauro et al, (2015). A Metodologia Das Maratonas de Programação Em Um Projeto de Extensão: Um Relato de Experiência. CBIE (2015).

Oliveira, Armanda M, Rodrigues, Rodrigo L, Garcia, Vinicius C, (2012). Um Mapeamento Sistemático Para Problem Based Learning Aplicado À Ciência Da Computação. WIE (2012).

Prince, M. (2004). Does active learning work? A review of the research. JOURNAL OF ENGINEERING EDUCATION, 93(3).

Souza, Marcella S. C., Costa, Felipe A. M., Silva, Victor Luan. Terra, Daniela Costa (2016). Lord of Code: uma ferramenta de apoio ao ensino de programação. In: Anais do XXVII do SBIE.

Slavin, R. E. (1980). Cooperative learning. Review of Educational Research.

Williams, L. and Kessler, R. (2002). Pair Programming Illuminated. Addison-Wesley Longman Publishing Co., Inc., Boston, MA, USA. 